\title{
SOBRE LA HISTORIA DE LA FILOSOFÍA DE LA CIENCIA. A PROPÓSITO DE UN LIBRO DE C. ULISES MOULINES
}

\author{
C. Ulises Moulines, El desarrollo moderno de la filosofía \\ de la ciencia (1890-2000), trad. Xavier de Donato, Instituto \\ de Investigaciones Filosóficas-UNAM, México (Filosofía \\ de la Ciencia), 2011, 186 pp.
}

Alejandro Cassini

CONICET-Universidad de Buenos Aires alepafrac@yahoo.com.ar

\section{Consideraciones generales}

La filosofía de la ciencia, entendida como una disciplina autónoma, es una creación reciente que sólo es claramente reconocible desde mediados de la década de 1930. A partir de entonces se ha desarrollado rápidamente, tanto que ya resulta imposible de abarcar para un único filósofo. Como consecuencia de ese desarrollo se ha ramificado y fragmentado en múltiples especialidades. Todo el que haya enseñado esta disciplina sabe bien cuán difícil es exponer esta ya compleja historia de una manera sintética, a la vez precisa y asequible a los que se inician en ella. Por esta razón resulta bienvenida la publicación de una obra de C. Ulises Moulines dedicada precisamente a este tema. ${ }^{1}$

Todavía no se ha publicado una historia detallada del surgimiento y el desarrollo de la filosofía de la ciencia como disciplina académica a lo largo del siglo XX. ${ }^{2}$ Es de presumir que una obra así sería muy voluminosa. El libro de Moulines presenta una síntesis de este proceso, necesariamente selectiva en contenidos, autores y bibliografía, y, sin embargo, consigue transmitir una gran cantidad de información en un espacio relativamente breve. Contiene, sin duda, algunas generalizaciones y simplificaciones que admitirían mayores matices pero que son imprescindibles en una síntesis de esta naturaleza. Es una

${ }^{1}$ Se trata de un libro publicado por Moulines en lengua alemana (Moulines 2008), el cual, a su vez, es una versión ampliada de otro libro publicado originalmente en francés (Moulines 2006). De acuerdo con lo que se indica en el prólogo, la traducción española parece haberse hecho sobre el texto francés, agregándosele luego los parágrafos nuevos de la edición alemana.

${ }^{2}$ Entre las obras generales publicadas con anterioridad a la que aquí se reseña pueden citarse Oldroyd 1986, Gower 1997 y Losee 2001. 
obra muy didáctica, clara y bien escrita; no obstante, nunca pierde el rigor necesario en la exposición de cada tema. También es una obra de madurez intelectual, que revela un profundo conocimiento de la filosofía de la ciencia. Su objetivo, ampliamente satisfecho por los resultados obtenidos, es presentar al lector no especialista un panorama de la filosofía general de las ciencias empíricas desde un punto de vista histórico. El libro de Moulines puede usarse como complemento de un curso universitario de filosofía de la ciencia, aunque por su extensión y tratamiento de los temas resultará insuficiente como libro de texto y deberá completarse con otras fuentes. ${ }^{3}$

En las primeras líneas del prólogo (p. 5), Moulines aclara que su obra es el resultado de una selección personal de los temas que considera más importantes. Así, deja voluntariamente de lado tanto la filosofía de la matemática, que tiene una historia larga, compleja y bastante autónoma, como la filosofía especial de la ciencia, esto es, la filosofía de las ciencias particulares, como la física, la biología o las ciencias sociales. Precisamente, la filosofía especial de las ciencias es la que más se ha desarrollo en las últimas décadas del siglo pasado, pero su inclusión habría complicado y extendido la obra, quitándole su carácter introductorio. La filosofía general de la ciencia se ha ocupado tradicionalmente de problemas comunes a un amplio grupo de ciencias, sobre todo de las llamadas ciencias naturales, ${ }^{4}$ entre ellos, la estructura y la dinámica de las teorías científicas, así como la explicación y la confirmación de hipótesis. Estos cuatro son los problemas principales, aunque no los únicos, que la obra de Moulines tiene en cuenta. Todos ellos pertenecen a lo que tradicionalmente se ha llamado "el contexto de justificación" de la ciencia, según la conocida dicotomía "descubrimiento-justificación" introducida por Reichenbach (1938), pero de hecho aceptada desde bastante tiempo antes. Son temas tradicionales que ya Popper había tratado, con diferente grado de detalle, en su primer libro publicado (Popper 1934). ${ }^{5}$ Moulines no

${ }^{3}$ El libro del autor en colaboración (Díez y Moulines 2008), en cambio, cumpliría muy bien esa función, ya que es el manual de filosofía general de la ciencia más completo que se haya escrito originalmente en lengua española hasta la fecha.

${ }^{4}$ No he podido determinar cuándo se presentó por primera vez de manera explícita la distinción entre filosofía general y especial de la ciencia, ni quién lo hizo. La referencia más antigua de que dispongo es Stegmüller 1979, de donde, posiblemente, la toma el propio Moulines. En la actualidad, en razón de la especialización y la fragmentación de la disciplina, ya no me parece que la distinción pueda trazarse con claridad. Incluso cabe preguntarse si todavía es posible la filosofía general de la ciencia. Me he ocupado de este problema en Cassini 2010.

${ }^{5}$ Con todo, el estudio sistemático de estos problemas no se produjo de manera 
menciona la rehabilitación del contexto de descubrimiento en la filosofía de la ciencia del último cuarto del siglo XX. Tampoco le dedica espacio a la filosofía de la experimentación, una temática nueva pero vigorosa, ni a la teoría de la confirmación, un tema clásico renovado en su vertiente bayesiana. De manera más general, no se ocupa de las prácticas científicas ni del estudio de la ciencia como proceso. Su enfoque es más cercano al de la filosofía tradicional de la ciencia, centrado casi exclusivamente en los productos de la actividad científica y, en particular, en las teorías.

En casi todos los temas que aborda, el libro de Moulines no se limita a una pura exposición, sino que las diferentes posiciones se someten a una breve pero penetrante crítica. Por cierto, el punto de vista del autor no es neutral y resultará evidente para cualquier lector atento: la concepción semántica de las teorías y, en general, los enfoques modelísticos de la filosofía de la ciencia tienen clara prioridad sobre otras perspectivas e incluso constituyen la piedra de toque con la cual se juzgan algunas posiciones alternativas.

\section{2. ¿Cuándo comenzó la filosofía de la ciencia?}

Toda periodización de un proceso histórico es esencialmente convencional y, como tal, sujeta a discusión. Sin embargo, necesitamos de alguna ordenación de los eventos en corrientes, etapas o fases de desarrollo para poder comprenderlos. Moulines propone distinguir cinco fases en el desarrollo de la filosofía de la ciencia del siglo XX (pp. 17-18). ${ }^{6}$ La primera de ellas es la llamada fase de germinación o preformación, entre 1890 y 1918. La segunda es la fase de eclosión, entre 1918 y 1935. La tercera es la fase clásica, entre 1935 y 1970. La cuarta es la fase historicista, entre 1960 y 1985. La quinta es la fase modelista, entre 1970 y 2000, que continúa a comienzos del siglo XXI hasta nuestros días. Los nombres y las fechas límite son aproximados y admiten matices y excepciones, como el autor reconoce (p. 31), por lo que no capturan todas las corrientes y tendencias, algo que sería tal vez imposible de encuadrar en un único esquema. Por último, no se trata de una periodización en sentido estricto, ya que las tres últimas fases se superponen en lapsos de diez y quince años. Posiblemente, por esa razón las denomina "fases" y no "periodos".

simultánea. Así, por ejemplo, el de la estructura de las teorías precedió al de la explicación científica, y éste, a su vez, al de la dinámica de las teorías. No obstante, todos ellos se hallaban ya bien establecidos en la agenda de la filosofía clásica de la ciencia antes de que ésta declinara a comienzos de la década de 1960.

${ }^{6}$ Cuando no hay otra indicación, los números de página entre paréntesis se refieren a la traducción española objeto de este estudio crítico. 
Es evidente que podemos encontrar reflexiones filosóficas acerca del conocimiento que hoy llamamos científico en muchos de los grandes clásicos de la tradición filosófica desde Aristóteles, en particular en los Analíticos segundos. ${ }^{7}$ Pero ello pertenece a la extensa prehistoria de la disciplina, que va desde los orígenes griegos hasta el siglo XIX. Se ha sostenido a veces que el libro de John Herschel A Preliminary Discourse on the Study of Natural Philosophy, publicado en 1830, es la primera obra específicamente dedicada a la filosofía de la ciencia y hay buenas razones para considerarlo así. Sin embargo, a lo largo del siglo XIX la filosofía de la ciencia todavía no está constituida como disciplina autónoma. No hay, por ejemplo, filósofos profesionales especialistas en ella. La mayoría de los clásicos precursores, como Herschel (1830), Whewell (1840), Jevons (1874) y Pearson (1892), actualmente poco leídos, son científicos y no filósofos. Además, la filosofía de la ciencia del siglo XIX todavía no está separada de la lógica y, como ocurre en Mill (1843) y mucho después de él, se concibe como una rama de la lógica, como lógica aplicada o metodológica. La situación todavía es así a principios del siglo XX, donde las figuras más significativas, como Mach, Duhem, Poincaré y tantos otros, son primariamente científicos o historiadores de la ciencia, pero no filósofos. La mayoría de las obras publicadas en torno a la década de 1920 que hoy podemos identificar como específicamente dedicadas a la filosofía general de la ciencia, como las de Campbell (1920 y 1921), Bridgman (1927) o Weyl (1927), todavía están escritas por científicos activos en sus respectivas disciplinas, generalmente la física. Uno de los primeros manuales reconocibles como introducciones a la filosofía de la ciencia escritos por filósofos, el de Cohen y Nagel (1934), no lleva esa denominación en su título y trata el tema, siguiendo la tradición de Mill, como lógica aplicada o metodología de la ciencia. En otros casos, como en las obras de Schlick (1918) o de Carnap (1928), filósofos que provienen de la ciencia, pero que ya no la ejercen, la filosofía de la ciencia todavía está integrada en la teoría del conocimiento empírico en general. De manera significativa, la revista creada por los positivistas lógicos se llamó Erkenntnis. Creo que hay un sentido bastante claro del término según el cual la filosofía de la ciencia como disciplina profesional y autónoma todavía no se encuentra constituida antes de 1935, año en el que Moulines señala el comienzo de la fase clásica.

\footnotetext{
${ }^{7}$ Las siguientes y todas las afirmaciones a lo largo del trabajo en las que no se hace referencia explícita a la obra de Moulines expresan las opiniones de quien esto escribe.
} 
Una disciplina académica profesionalizada, en cualquier ámbito del conocimiento, nunca emerge completa de una manera repentina. Por lo general, es el resultado de un proceso de formación a veces bastante extenso y muy a menudo de carácter gradual. El hecho de que una determinada disciplina se encuentra ya constituida puede reconocerse por medio de diferentes criterios externos, que pueden considerarse como indicadores sociológicos. Aquí consideraré los seis que me parecen más importantes. No hay un orden lógico ni temporal entre ellos, que de hecho suelen presentarse agrupados de distintas maneras. El primer indicador es la identificación de la disciplina mediante un nombre común específico y reconocido por todos sus practicantes. En muchos casos, el nombre llega tardíamente. No conozco libros o revistas que lleven el título explícito de filosofía de la ciencia antes de mediados de la década de 1930. El nombre empieza a utilizarse sistemáticamente a partir de la fundación de la revista Philosophy of Science en 1934. Si tuviera que elegir una fecha como comienzo de la disciplina, elegiría ésta. El segundo indicador es precisamente la aparición de revistas profesionales especializadas en la disciplina en cuestión. Hasta 1934 existía Erkenntnis, cuyo contenido es reconociblemente de filosofía de la ciencia, pero también incluye muchos otros temas como la lógica, la filosofía del lenguaje y la metafísica. El tercer indicador es la puesta en marcha de congresos específicamente dedicados a la temática de esta disciplina. Los congresos dedicados a la "filosofía científica" y a la "unidad de la ciencia" que organizaron los positivistas lógicos entre 1935 y 1940 caen muy cerca de satisfacer este criterio, pero todavía no lo cumplen plenamente, tal vez por falta de una identificación común. El cuarto indicador es la existencia difundida de cátedras dedicadas a la disciplina y, correlativamente, de su presencia sistemática en los planes de estudio de las respectivas carreras. Éste es un proceso que se debe investigar y no tengo información fiable al respecto, pero todo indica que, salvo cátedras aisladas o efímeras, la presencia de la filosofía de la ciencia como parte de los planes de estudio de las carreras de filosofía, como ocurre hoy en día y desde hace varias décadas, es un proceso lento y gradual, que no se consuma en los países de Europa o en Estados Unidos hasta finales de la década de 1940 o incluso después, mientras que en América Latina no lo hace por lo menos hasta la década de 1960. El quinto indicador que señalaré es la publicación de libros de texto dedicados a la enseñanza elemental y/o avanzada de la disciplina, generalmente agrupados en colecciones de una misma editorial. Ya señalé que a mediados de la década de 1930 todavía no 
existen tales libros de texto, a diferencia de, por ejemplo, la lógica matemática, que ya contaba con varios. Sería interesante determinar cuál fue el primero que llevó en su tapa el título filosofía de la ciencia sin otro aditamento. Ese hecho constituye en mi opinión un indicio claro de que una disciplina se reconoce como tal sin necesidad de subtítulos u otras aclaraciones. El sexto indicador es la existencia de sociedades o asociaciones profesionales destinadas a promover una disciplina específica. A menudo tal sociedad es la misma que edita alguna revista profesional u organiza los congresos de la disciplina, por lo que éste no siempre es un criterio independiente. El Círculo de Viena, al menos en su faceta pública desde 1930, se halla muy cerca de satisfacer este criterio. La denominación de "filosofía científica", nombre que se incorporó al primer congreso para la unidad de la ciencia, celebrado en París en 1935, captura mejor que la etiqueta más restrictiva de "filosofía de la ciencia" el alcance de los intereses iniciales de Neurath y otros miembros del Círculo. ${ }^{8}$ La idea todavía persiste en el título de la última obra que Reichenbach publicó en vida, en una fecha tan tardía para el desarrollo del empirismo lógico como 1951.

Queda, sin duda, mucho por investigar en lo relativo a cada uno de estos seis indicadores. Con todo, la información parcial y fragmentaria de que dispongo apunta nítidamente a la conclusión de que la filosofía de la ciencia como disciplina académica reconocida y como especialidad profesional de un grupo de filósofos todavía no se encontraba constituida antes de mediados de la década de 1930, aunque estaba evidentemente en un proceso acelerado de formación. Después de 1936, la disciplina ya es claramente reconocible, pero en esa fecha el Círculo de Viena ya se había desintegrado (el asesinato de Schlick, el 22 de junio de 1936, representa, sin duda, su acta de defunción). ${ }^{9}$

\section{De los precursores a los clásicos}

El capítulo que Moulines consagra a la fase de preformación de la filosofía de la ciencia es muy breve (pp. 19-29) y se propone señalar

${ }^{8}$ Estos intereses incluían, por ejemplo, una reforma lingüística y pedagógica de la propia ciencia, objetivos que luego la filosofía clásica de la ciencia abandonó, o, al menos, dejó en un segundo plano.

${ }^{9}$ La obra enciclopédica de Stadler (1997) contiene el índice completo de los ocho volúmenes de Erkenntnis (1930-1940), así como los programas de todas las contribuciones de los cinco Congresos para la Unidad de la Ciencia (1935-1940). El contenido disciplinar de la filosofía de la ciencia es claramente identificable en ambos casos, aunque el nombre todavía no está en uso. 
la presencia de algunas semillas que luego germinarían en las fases siguientes. Una de ellas es el programa fenomenista de Mach, al que Moulines asigna gran importancia. Uno de los legados de este programa es una reconstrucción de la física sin términos teóricos sospechosos de ser puramente metafísicos, como "espacio absoluto" o "fuerza". Mach intentó hacerlo con la mecánica newtoniana, y, aunque su programa de eliminación o reducción de los términos teóricos quedó inconcluso, se retomó durante las primeras etapas del positivismo lógico. El otro legado de Mach es una reconstrucción de todo conocimiento empírico sobre bases fenomenistas, esto es, sobre sensaciones o datos elementales de los sentidos. Russell retoma este programa en Our Knowledge of the External World de 1914, pero es Carnap quien lo lleva cabo por primera vez de manera rigurosa en el Aufbau de 1928. Allí se aplican exitosamente los métodos de análisis lógico que Russell mismo había perfeccionado, pero no había aplicado de manera sistemática, como lo hizo Carnap. El programa fenomenista culmina, sin duda, con la gran obra de Goodman, The Structure of Appearence, publicada en 1951, que curiosamente Moulines no menciona. En la segunda mitad del siglo XX el programa fenomenista fue perdiendo la mayor parte de su atractivo, al menos en la filosofía de la ciencia, una vez que se reconoció la imposibilidad de reducir a conceptos observacionales los conceptos teóricos de la física, incluso los conceptos métricos más elementales, como el de longitud.

La otra posición influyente de esta primera etapa la constituyen, según Moulines, el instrumentalismo y el convencionalismo, dos doctrinas estrechamente asociadas y a veces indistinguibles, que tienen sus mayores representantes en Poincaré (1902) y Duhem (1906). Esta tendencia tuvo fuera de Europa una influencia más tardía, pero más profunda, a partir de la obra de Quine y de las tardías traducciones de Duhem al inglés en la década de 1950. Habría sido justo, en mi opinión, que Moulines mencionara aquí el ficcionalismo de Vaihinger, expresado en su influyente Die Philosophie des Als Ob, que tuvo diez ediciones, sucesivamente aumentadas, entre la primera de 1911 y la última de 1927. El ficcionalismo experimentó una sorprendente resurrección a fines del siglo XX, sobre todo a partir del artículo de Arthur Fine (1993) que lo insertó en la discusión acerca de la naturaleza de los modelos y las idealizaciones en la ciencia.

La segunda fase, la de eclosión, es para Moulines, esencialmente la del surgimiento y la consolidación del positivismo lógico, particularmente en el Círculo de Viena y otros lugares de Europa central, 
como Berlín, Praga o Cracovia (pp. 31-48). Las características fundamentales de este movimiento, al que es preferible llamar "empirismo lógico", se encuentran expresadas en los dos términos que componen su nombre. Por una parte, una orientación empirista, que tiene muy poca relación con el positivismo francés de Comte (quien, por su parte, era muy poco empirista), sino que fue heredada principalmente de Mach y Russell. Por otra parte, un interés decisivo en la lógica matemática y los métodos formales de análisis como herramientas indispensables para la filosofía de la ciencia, e incluso para la filosofía en general, descendiente de la posición de Frege. Esta parte de la historia es bien conocida y Moulines la expone de manera clara y sintética. Con todo, el autor es consciente de que no todas las tendencias de esa época se dejan atrapar por este movimiento. La epistemología francesa, orientada de manera predominante hacia un enfoque histórico de los temas y problemas, es una de ellas. Moulines señala las obras de Bachelard a partir de la década de 1930 y de Canguilhem desde la década siguiente, como los hitos que moldearon esta "excepción francesa" (p. 31), como la llama. Podría decirse, sin embargo, que esa orientación histórica ya era evidente mucho antes, con la obra de autores como Meyerson (1908), cuyos libros merecen citarse como una contribución permanente a la filosofía de la ciencia del siglo XX.

La fase clásica de la filosofía de la ciencia, a partir de 1935, ya no se desarrolla en lengua alemana, sino inglesa, como consecuencia de la emigración de las principales figuras de Alemania y Europa central (Carnap, Reichenbach, Popper, Hempel y muchos otros) debido a la hegemonía del nazismo (pp. 49-82). Moulines sostiene que hacia esta época se produjo la caída de dos pilares del empirismo lógico original: el verificacionismo y el reduccionismo (pp. 49 y 57). En buena medida esto fue el producto de críticas internas, como las realizadas por Neurath, Popper y Carnap a la indubitabilidad y la justificación inmediata de los enunciados básicos. Estas críticas tuvieron consecuencias de largo alcance. Ante todo, en lugar del verificacionismo, Reichenbach y Carnap adoptaron el probabilismo, según el cual el conocimiento científico no es conocimiento verificado, sino meramente probable. El probabilismo condujo, por su parte, ya en la década de 1940, al desarrollo de la teoría cualitativa de la confirmación de Hempel (1945) y a la lógica inductiva cuantitativa de Carnap (1950). Ambos programas hallaron a su vez dificultades técnicas, que en las décadas siguientes les fueron quitando su atractivo original. Por otro lado, en lugar de intentar definir explícitamente los términos teóricos mediante términos observacionales, Carnap (1936-1937) optó por las 
llamadas "definiciones parciales" mediante reglas de correspondencia. Hacia mediados de la década de 1950 la concepción clásica de las teorías alcanzó su formulación final con la llamada "teoría de los dos niveles" (p. 63) de Hempel (1952) y Carnap (1956). De acuerdo con esta posición, una teoría empírica es un sistema axiomático formal globalmente interpretado mediante reglas de correspondencia para algunos de los términos teóricos de su vocabulario. De esta manera se termina por reconocer la imposibilidad de eliminar los términos teóricos del vocabulario de la ciencia. Sin embargo, el debate sobre la referencia de tales términos continuó por muchas décadas.

Moulines dedica algunas páginas interesantes a la oración de Ramsey (pp. 67-70) que de alguna manera parece mostrar que los términos teóricos son a fin de cuentas eliminables. Supongamos que una teoría se compone de un solo postulado teórico $\forall x\left(T x \rightarrow T^{\prime} x\right)$ y de una sola regla de correspondencia $\forall x(T x \rightarrow O x)$ (donde $T$ y $T^{\prime}$ son términos teóricos y $O$ es un término observacional). Ésta es una teoría perfectamente aceptable según la versión final de la concepción clásica. La oración de Ramsey de esa teoría es: $\exists \phi \exists \psi \forall x((\phi x \rightarrow \psi x)$ $\&(\phi x \rightarrow O x))$. Aquí está claro que las variables cuantificadas $\phi$ y $\psi$ son de segundo orden, algo que en la formalización que emplea Moulines (p. 68) no es evidente. La oración de Ramsey de una teoría que contiene términos teóricos no es lógicamente equivalente a ella, pero es empíricamente equivalente en el sentido de que toda consecuencia observacional de una teoría (posiblemente, junto con ciertas hipótesis auxiliares, condiciones iniciales y condiciones de contorno) es también una consecuencia observacional de su oración de Ramsey. Moulines señala con razón que esta estrategia tiene un "sabor instrumentalista" (p. 69) en la medida en que parece suponer que la única función de los términos teóricos es permitir la deducción de predicciones, o, a lo sumo, la conexión entre diferentes enunciados de observación. El filósofo realista replicaría inmediatamente que en la oración de Ramsey la eliminación de los términos teóricos es puramente sintáctica y que el problema semántico de la referencia de estos términos reaparece en cuanto se pregunta por la interpretación de esa oración, en particular, por el dominio de las variables de segundo orden que se han cuantificado existencialmente. No es de extrañar, entonces, que la cuestión de la referencia de los términos teóricos haya persistido después de que la obra de Ramsey fuera redescubierta en la década de 1950. Como afirma Moulines (p. 70), la controversia continúa abierta en nuestros días. Y con razón, cabría agregar, ya que ningún método de "eliminación funcional" de los 
términos teóricos, como la oración de Ramsey o el teorema de Craig (1953 y 1956) (al que Moulines no hace referencia, pero que sin duda merecía un lugar en esta historia), podrían resolverla ya que simplemente eluden el problema. Presuponen que la función de una teoría es sólo organizar los enunciados observacionales y permitir las inferencias entre tales enunciados. Dos teorías empíricamente equivalentes son igualmente capaces de cumplir esta función, cualesquiera que sean sus postulados teóricos. De allí a prescindir de los términos teóricos hay apenas un paso, que todo empirista parecía dispuesto a dar en ese momento. Pero, como ya lo señaló Hempel en su clásico trabajo crítico (Hempel 1958), las teorías que se obtienen mediante los métodos de Craig y de Ramsey tienen muchas desventajas: son menos simples, menos explicativas y menos fértiles desde el punto de vista heurístico que las teorías formuladas con términos teóricos, por lo que en la práctica la utilidad de dichos métodos es muy escasa.

El artículo de Quine "Two Dogmas of Empiricism" (1951), cuyo efecto Moulines califica de "demoledor" (p. 72), propinó uno de los mayores golpes a la filosofía clásica de la ciencia. Quine sembró serias dudas sobre la distinción entre enunciados analíticos y sintéticos, aunque en modo alguno probó la imposibilidad de trazarla. Por otra parte, introdujo en la filosofía anglosajona el holismo epistemológico de Duhem, fundado en una concepción deductivista de la confirmación, en un momento en que era casi desconocido. Quine generalizó a todo el conocimiento el holismo restringido de Duhem, limitado a las hipótesis de la física teórica. Además, sacó consecuencias bastante radicales de este holismo generalizado, entre ellas, la célebre tesis de la subdeterminación de toda teoría por la experiencia, que nunca llegó a formular de manera precisa. Esta tesis afectaba tanto al inductivismo de Carnap como al falsacionismo de Popper y no tuvo una réplica satisfactoria por parte de ninguno de los dos. En cierto sentido puede decirse que en ese momento se inició la crisis terminal de la filosofía clásica de la ciencia.

\section{La irrupción de la historia}

Se ha dicho muchas veces que el positivismo lógico y la filosofía clásica de la ciencia ignoraban que todo conocimiento, y la ciencia en particular, tiene una historia. El aserto es exagerado, pero tiene un grano de verdad. Moulines califica de "ahistórico" el enfoque de los filósofos clásicos (p. 83) haciéndose eco de esta interpretación tradicional. Los filósofos clásicos de la ciencia, en efecto, se preocuparon esencialmente por cuestiones relacionadas con el contexto 
de justificación de las teorías y relegaron los factores históricos al dominio del contexto de descubrimiento, el cual, por el hecho de no estar sujeto a reglas lógicas, caía fuera de los límites de la filosofía de la ciencia. El enfoque clásico estaba basado en el análisis lógico y era de carácter fundamentalmente normativo, centrado en la reconstrucción racional de teorías, y entre ellas, casi exclusivamente las de la física contemporánea. La situación cambió de manera más o menos rápida hacia finales de la década de 1950, con obras como Patterns of Discovery de Hanson (1958), la cual en mi opinión señala el comienzo del enfoque historicista en la filosofía de la ciencia. Moulines menciona a Hanson y Toulmin como "precursores" (p. 84), pero creo que merecían algún comentario más caritativo. La obra de Hanson, por ejemplo, rehabilitó el contexto de descubrimiento como campo accesible al análisis filosófico, y con ello dio entrada a todos los factores históricos y "externos", conscientemente relegados por los empiristas lógicos. Además, introdujo la noción de "carga teórica de la observación", una idea que procedía de Duhem, pero que había pasado casi inadvertida. Con ello cuestionó uno de los dogmas esenciales del empirismo lógico: el de la neutralidad de la base empírica de las teorías y su consiguiente estabilidad frente al cambio teórico. Feyerabend primero y luego Kuhn sacaron consecuencias radicales de esta tesis y pusieron en duda la posibilidad de contrastar teorías rivales mediante la experiencia (otra idea que también había sido anticipada por Duhem en su crítica de los experimentos cruciales). Toulmin, por su parte, ya había escrito uno de los primeros libros de filosofía general de la ciencia que se apartaban de las líneas generales del empirismo lógico y de la filosofía clásica de la ciencia (Toulmin 1953), y luego anticipó muchas de las ideas de Kuhn sobre los paradigmas y las revoluciones científicas, utilizando una terminología diferente $\mathrm{y}$, sin duda, menos atractiva y exitosa que la de Kuhn (Toulmin 1961).

Moulines expone sucesivamente las ideas de Kuhn, Feyerabend, Lakatos y Laudan, a quienes considera los representantes principales de la corriente historicista (pp. 83-102). Difícilmente se podría disentir en este punto. Cabe observar, sin embargo, que no todos los filósofos de la ciencia se volvieron historicistas después del impacto de la obra de Kuhn. Ante todo, muchos filósofos del periodo clásico, como Popper (1994), lo criticaron sin contemplaciones; otros, como Hempel (2001), recibieron su influencia, pero no aceptaron sus consecuencias más radicales, como la tesis de la inconmensurabilidad. Por otra parte, diversos filósofos de la ciencia contemporáneos de los historicistas, como Patrick Suppes, trabajaron paralelamente sin 
preocuparse mucho por la historia de la ciencia ni por los modelos de cambio científico de los historicistas. ${ }^{10}$ Hubo durante esta fase innumerables críticas a Kuhn, pero la más lograda, la de Scheffler (1967), todavía se lee con provecho y merece registrarse. El historicismo debe considerarse, entonces, como una corriente mayoritaria y sumamente influyente durante las décadas de 1960 y 1970, pero en modo alguno como la orientación unánime de los filósofos de la ciencia de este periodo.

La influencia del historicismo fue mayor en campos como la historia, la sociología de la ciencia y las ciencias sociales que en la propia filosofía de la ciencia. Moulines señala acertadamente que la tesis de la inconmensurabilidad de Kuhn se interpretó generalmente "como una forma radical de relativismo epistemológico" (p. 93) y que algunos sociólogos de la ciencia sacaron de allí la conclusión de que la filosofía general de la ciencia se había vuelto "obsoleta". Kuhn mismo siempre rechazó esta clase de interpretaciones y en sus últimos trabajos luchó en vano contra ellas. Paradójicamente, la obra de Kuhn influyó en aquellas disciplinas que él mismo había excluido de su modelo de cambio científico, como la historia o la sociología, a las que consideraba todavía en una etapa precientífica o preparadigmática.

Moulines termina su presentación de la fase historicista con una exposición muy crítica, bajo el rótulo de "relativismo socio-epistémico" (p. 102), de las ideas del llamado "programa fuerte" de la Escuela de Edimburgo de Bloor y Barnes, y de otros autores como Latour y Knorr-Cetina, indudablemente influidos por aquéllos. Él considera estos desarrollos sociológicos como una deriva extrema de algunas tesis de Kuhn y Feyerabend. Hay otros antecedentes, por cierto, como la sociología clásica del conocimiento, de claras raíces marxistas. Moulines afirma que el relativismo socio-epistémico, según el cual la verdad y la justificación de una teoría científica son relativas a una cultura determinada, es una tesis esencialmente vaga y mal definida (p. 107). Como consecuencia de ello difícilmente se la puede criticar. Por otra parte, está expuesta al peligro de autorrefutación, ya que para ser consecuente debería ser autorreferencial. Éste es el argumento tradicional, desde que Platón lo usó contra los sofistas, pero que ahora los relativistas del programa fuerte están dispuestos a aceptar. Moulines concluye, entonces, que el relativismo fuerte es "tan sólo una manifestación suplementaria, particularmente desafortunada y

\footnotetext{
${ }^{10}$ Suppes 2002 resume toda su obra desde la década de 1960 y reproduce de manera casi literal muchos de sus trabajos clásicos, entre ellos, Suppes 1967.
} 
grotesca, del espíritu relativista que invade ciertos departamentos de ciencias humanas en las universidades occidentales" (p. 107). Este juicio me parece excesivo. Aunque el relativismo extremo de algunos sociólogos de la ciencia sea evidentemente inaceptable, los estudios sociales de la ciencia (por ejemplo, Pickering 1992) han tenido, en mi opinión, el mérito de señalar que la ciencia es fundamentalmente una actividad humana inmersa en un sistema cultural más amplio y de poner de manifiesto una dimensión, la de las prácticas científicas, a la que la filosofía de la ciencia había prestado escasa atención casi hasta finales del siglo XX.

Moulines no traza un balance del historicismo, pero constata que su influencia mengua después de 1980 y que los filósofos de la ciencia actuales, salvo excepciones, muestran poca conexión con los filósofos historicistas (p. 108). Yo sugeriría que hay dos razones para este declive. La primera es que las principales tesis historicistas, en particular las de Kuhn, son irremediablemente vagas. Por tanto, resultan muy difíciles de desarrollar y de aplicar a casos concretos. En su libro fundamental Kuhn (1962) no proporcionó ningún criterio para reconocer cuándo un cambio teórico es una revolución científica. Posteriormente sostuvo, replicando a sus críticos, que en la historia de la ciencia hay revoluciones mayores y menores, y admitió que en muchos casos él mismo no era capaz de determinar si un cambio teórico específico constituía o no una revolución científica (Kuhn 1970). ${ }^{11} \mathrm{La}$ segunda razón es que los modelos de cambio científico propuestos por los historicistas son demasiado generales y poco articulados, de manera que cualquier investigación histórica de la ciencia encuentra rápidamente contraejemplos, o bien casos grises y dudosos que no se pueden acomodar con facilidad dentro del modelo general. Algo así le sucedió probablemente al propio Kuhn en su trabajo como historiador de los orígenes de la mecánica cuántica. No ha dejado de señalarse que en su obra historiográfica más importante (Kuhn 1978) no emplea sus principales categorías epistemológicas ni cita su libro sobre las revoluciones científicas (posteriormente, Kuhn 1984, intentó explicar estos hechos). Por otra parte, ésta es tal vez la obra de Kuhn más apreciada en la actualidad, ya que, luego de una recepción crítica, se ha revalorizado y se considera que ha hecho aportes novedosos

${ }^{11}$ En sus trabajos de la década de 1980 y comienzos de la de 1990 (recopilados en Kuhn 2000) sostuvo que cuando se produce un cambio teórico revolucionario, la inconmensurabilidad entre teorías sucesivas se manifiesta en la aparición de estructuras taxonómicas o esquemas conceptuales incompatibles. Así, la presencia de estas taxonomías no homologables proporcionaría un indicador para reconocer la existencia de las revoluciones científicas. 
para comprender el papel de Planck y Einstein en un proceso al que todo el mundo calificó de revolucionario.

Hay, con todo, una herencia indudable de la fase historicista que permanece en la filosofía de la ciencia actual. Se trata del valor que casi de manera unánime se concede a los estudios de casos, ya sean de la historia de la ciencia lejana o contemporánea, como evidencia a favor de una determinada hipótesis epistemológica. Casi ningún filósofo de la ciencia actual se adhiere a una manera puramente normativa y ahistórica de practicar su disciplina. Aun cuando trate temas tradicionales y muy amplios de filosofía general de la ciencia, como la explicación y la confirmación de teorías, intentará casi siempre mostrar que sus ideas se adecuan a casos reales de investigación científica. Ya nadie se conforma con apelar a las remanidas teorías $T_{1}$ y $T_{2}$ (que tanto desagradaron siempre al científico practicante), ni con el recurso a ejemplos artificiales o ficticios, ni a experimentos mentales imaginados por los filósofos. Casi toda obra seria de filosofía de la ciencia, desde hace al menos tres décadas, incluye el análisis más o menos detallado de al menos algún caso histórico de investigación científica y, muy probablemente, también el de algún caso significativo de cambio teórico. En ello radica, en mi opinión, el aporte positivo y permanente de los filósofos historicistas. Aunque ya nadie persigue el ideal de formular un modelo general de cambio científico, gracias a la obra de Kuhn y sus contemporáneos casi todos los filósofos de la ciencia se han vuelto sensibles a la historia de la ciencia y reconocen su relevancia para la filosofía de la ciencia.

\section{5. ¿Modelos y sólo modelos?}

El último capítulo de la obra, que lleva por título "Concepciones modelísticas y emparentadas (1970-2000)", es el más extenso (pp. 109165). Es también el que me ha parecido menos logrado. Creo que está excesivamente sesgado hacia la llamada concepción semántica de las teorías, a la que Moulines dedica la mayor parte de las páginas (111-144), y de la que ofrece una presentación muy completa y precisa, sobre todo de la corriente estructuralista (pp. 134-144), una corriente cuya influencia, en mi opinión, ha sido menor que la de otras variantes de la familia semanticista (como, por ejemplo, las de van Fraassen y Giere). Se comprende la elección, pues el propio autor es un miembro distinguido de la familia semanticista y ha contribuido a formular una posición, el estructuralismo metateórico, al que considera claramente superior a otras concepciones semánticas. 
La elección de este hilo conductor presenta, en mi opinión, varios inconvenientes. En primer lugar, uno de tipo cronológico. La concepción semántica de las teorías se origina a principios de la década de 1950, con los trabajos de McKinsey y Suppes, ${ }^{12}$ esto es, mucho antes del comienzo de la fase historicista, que Moulines establece en 1960. Si hasta ahora había una superposición parcial entre las diferentes fases, cosa razonable, ahora el orden se trastoca, ya que la concepción semántica resulta contemporánea de la fase clásica de las teorías. Algo parece funcionar mal en esta periodización. En verdad, el surgimiento y desarrollo de la concepción semántica ocurrió en paralelo con las fases clásica e historicista (por ejemplo, en Suppes 1957 y 1967). No es, en sentido estricto, una creación propia de las dos o tres últimas décadas del siglo XX, cosa que el propio Moulines reconoce (p. 112). La llamada fase modelística, entonces, no debería considerarse como la fase más reciente en el desarrollo de la filosofía de la ciencia, sino como una de las tantas corrientes filosóficas que evolucionaron a lo largo de la segunda mitad del siglo XX.

En segundo lugar, la elevación del concepto de modelo al primer plano de la filosofía de la ciencia efectivamente ocurrió a partir de la década de 1980, pero luego el estudio de los modelos se independizó de la concepción semántica de las teorías y se condujo por sí mismo. De hecho, muchos autores que en la actualidad se dedican a analizar los modelos científicos no se adhieren a la concepción semántica de las teorías, e incluso han dejado de emplear el concepto mismo de teoría sustituyéndolo por el de modelo. Algunos han afirmando de manera explícita que son los modelos y no las teorías los auténticos vehículos del conocimiento científico (así, por ejemplo, Morgan y Morrison 1999 y Suppe 2000).

En tercer lugar, una gran parte de los temas y problemas discutidos por los filósofos de la ciencia de las dos últimas décadas del siglo XX no tienen relación directa con el concepto de modelo y a veces son completamente independientes de él. Entre ellos podrían señalarse la filosofía de la experimentación, la lógica del descubrimiento, el antirrealismo científico, la "desunidad" de la ciencia, la teoría bayesiana de la confirmación y la mayoría de las concepciones de las leyes naturales y la explicación científica. Moulines lo reconoce

${ }^{12}$ Donde se presenta la axiomatización de la mecánica clásica de partículas y la mecánica relativista de partículas mediante la introducción de un predicado conjuntista (McKinsey, Sugar y Suppes 1953, y Rubin y Suppes 1954, respectivamente). Esos trabajos se difundieron entre los filósofos recién a partir de la obra de Suppes 1957. 
parcialmente cuando expone las teorías mecánico-causal y unificacionista de la explicación y admite que "estos nuevos enfoques no tienen que ver con la última fase de la historia de nuestra disciplina - el modelismo - " (p. 152). Lo mismo podría decirse de la discusión del realismo científico, tema al que se le dedica el último parágrafo del capítulo (pp. 158-165). En suma, creo que la elección de la noción de modelo, y sobre todo de la concepción semántica de las teorías, como eje de la última fase constituye un criterio demasiado estrecho como para capturar los desarrollos más importantes de la filosofía de la ciencia reciente.

De las muchas cuestiones que Moulines trata en este capítulo hay una que quisiera discutir con algún detalle. Se trata de la oposición entre la concepción sintáctica y la concepción semántica de las teorías, que, según creo, está mal concebida. Moulines insiste a lo largo de su libro en que la concepción clásica de las teorías empíricas (la de Carnap, Hempel y otros) es formalista y sintactista (pp. 109-110, entre otras). Para la concepción clásica una teoría empírica es la clausura deductiva de dos conjuntos de oraciones: el de los axiomas o postulados teóricos y el de las reglas de correspondencia. Pero estas últimas son precisamente reglas semánticas, cuya función principal es conferir significado a los postulados teóricos. Una teoría que contuviera sólo postulados teóricos sin interpretar no sería una teoría empírica, sino puramente formal. Así, la concepción clásica es completamente semántica desde el comienzo. El hecho de que la sintaxis y la semántica se especifiquen por separado, mediante conjuntos diferentes de oraciones, no implica que se trate de una concepción sintactista. Por consiguiente, la oposición entre una concepción semántica y una sintáctica no capta ninguna diferencia esencial. Es mejor distinguir entre una concepción de las teorías como conjuntos de proposiciones y otra que las concibe como colecciones de modelos. Aun así, la oposición no es completa, ya que todas las concepciones semánticas apelan a un elemento proposicional (bajo la forma de "hipótesis teóricas" o "aserciones empíricas") para relacionar los modelos con sus dominios de aplicación, por lo que una teoría no puede identificarse solamente con una colección de modelos.

Otras dos ideas que no me parecen acertadas respecto de la concepción clásica, pero que Moulines suscribe, son que ésta es una concepción lingüística o "enunciativa" de las teorías y que está ligada indisolublemente a la formalización en un lenguaje de primer orden. Dentro de la concepción clásica es posible distinguir claramente entre una teoría, concebida como un conjunto de proposiciones, y las diferentes formulaciones de esa teoría, realizadas mediante un conjunto 
de oraciones de un determinado lenguaje. Por otra parte, no creo que las teorías empíricas deban presentarse formalizadas en lenguajes de primer orden. Pueden formularse en cualquier lenguaje y, además, presentase de manera formalizada o no formalizada. Lo que les confiere su identidad es el conjunto de las proposiciones expresadas, pero no el lenguaje en el que se formulan. La limitación a los lenguajes de primer orden, más implícita que explícita en los autores clásicos, se debió al hecho de que éstos eran los que mejor conocían los lógicos y los filósofos de la ciencia hacia la década de 1930. Treinta años después, la situación ya no era la misma y nada obligaba a mantener esa limitación.

El problema principal de la concepción clásica no es que haya sido lingüística, ni sintactista, ni formalista, sino que nunca logró elucidar adecuadamente su aspecto semántico. El carácter de las reglas de correspondencia, concebidas como definiciones parciales de los términos teóricos, nunca estuvo claro: ¿eran definiciones estipuladas por convención o hipótesis empíricas que debían descubrirse en el mundo? Al parecer, resultaron una suerte de híbrido cuyo estatus analítico o sintético era particularmente oscuro. Por otra parte, el cuestionamiento de la distinción entre términos teóricos y observacionales, que popularizó Hanson, le quitó interés al proyecto de definir, aunque sólo fuera "parcialmente", una categoría, la de los términos observacionales, cuyos límites ya no podían trazarse claramente. Con todo, una concepción proposicional de las teorías, sin compromiso alguno con la distinción teórico-observacional ni con las reglas de correspondencia, me parece todavía una alternativa viable a la concepción semántica. Al menos, no la alcanzan las críticas usuales de los semanticistas a la concepción clásica, como las que Moulines expone. $^{13}$

Al comienzo del capítulo, Moulines se muestra más sensible a la diversidad que caracteriza a la filosofía de la ciencia desde la década de 1980. A partir de esa fecha se desarrollan muchas corrientes y tendencias, a veces de manera bastante independiente entre sí. Como en toda la filosofía actual, el "espíritu de escuela" se encuentra ausente y los diferentes autores que comparten una misma posición general frecuentemente tienen sólo un "aire de familia en común". El propio Moulines señala (p. 109) que es difícil encontrar características comunes a todos los filósofos de la ciencia de esta fase. Por otra parte, como también advierte, se trata de desarrollos en muchos

${ }^{13}$ Ésta es, por cierto, una afirmación que debe justificarse. Me ocupo de esta cuestión en Cassini 2009. 
casos demasiado recientes para cuya evaluación todavía carecemos de la perspectiva que confiere el paso del tiempo. Se pueden constatar dos tendencias generales, dice el autor, una es el antirrealismo y otra es el pluralismo metodológico (pp. 110-111). Como tendencias mayoritarias me parecen evidentes. Sólo cabría hacer una reserva para el uso del término "antirrealismo", que puede entenderse de dos maneras bastante diferentes.

Si se entiende en un sentido estrecho, como sinónimo del viejo término "instrumentalismo", que hace tiempo que nadie quiere reivindicar, la afirmación no es correcta. No todos los filósofos de la ciencia estarían dispuestos a sostener que las teorías o los modelos científicos carecen de valor de verdad y de carácter referencial ya que son sólo herramientas predictivas. Si, en cambio, se entiende en un sentido más amplio, como negación del realismo clásico, la aserción de Moulines es cierta. Prácticamente ningún filósofo de la ciencia de esta última fase estaría dispuesto a suscribir las condiciones tradicionales de verdad y referencia de acuerdo con las cuales las teorías científicas son verdaderas y todos sus términos descriptivos son referenciales. La más fuerte de las versiones actuales del realismo, la de Boyd (1983), debilita ambas condiciones, afirmando que las teorías son verosímiles o aproximadamente verdaderas y que la mayoría de sus términos son referenciales (en particular, los que pretenden referirse a entidades o propiedades inobservables, los viejos términos teóricos). Como posiciones alternativas surgieron los llamados "realismos debilitados", los cuales o bien renuncian a alguna de las dos condiciones (como hace Hacking con la condición de verdad), o bien las debilitan aún más que Boyd, admitiendo, por ejemplo, el fracaso de las teorías de la verosimilitud. La pregunta que cabe hacerse, y que no me parece una mera cuestión verbal, es cuánto puede debilitarse el realismo sin caer en alguna forma de antirrealismo. La línea que separa a los realismos debilitados de los antirrealismos parece muy tenue y borrosa, sobre todo cuando se renuncia a la verdad como objetivo de la ciencia.

El relato de Moulines es eminentemente clásico en su punto de vista. Tiene como presupuesto la idea de que los productos más característicos de la ciencia son las teorías, las cuales constituyen el auténtico vehículo del conocimiento científico. De allí la importancia que asigna a la concepción semántica de las teorías como alternativa a la concepción clásica, heredada de los empiristas lógicos. Indudablemente, durante la fase clásica de la filosofía de la ciencia la determinación de la estructura y, en menor medida de la dinámica, de las teorías científicas fue el problema fundamental. Pero las cosas cambiaron a partir de 1960. Los historicistas adoptaron para su análisis unidades 
epistemológicas más amplias que las teorías, como los paradigmas, los programas de investigación o las tradiciones de investigación, a las cuales las teorías estaban, por así decir, subordinadas. Después del historicismo, el supuesto de que las teorías deberían ser el objeto de estudio casi excluyente de los filósofos de la ciencia fue perdiendo terreno gradualmente. La atención se fue desplazando de las teorías a las prácticas científicas, o, en términos de Hacking (1983), de la representación a la intervención. La filosofía de la experimentación es un buen ejemplo de este cambio. El experimento, la medición y la observación son prácticas científicas que no producen teorías y que frecuentemente no están al servicio de ellas, sino que muestran un alto grado de autonomía frente al cambio teórico. Mientras la atención de los filósofos estuvo puesta sobre las teorías, estas prácticas científicas pasaron casi inadvertidas, salvo como proveedoras de evidencia para la contrastación. Cuando a mediados de la década de 1980 una diversidad de filósofos y sociólogos de la ciencia se volvieron hacia ellas, se abrió una nueva dimensión de la ciencia casi inexplorada.

El estructuralismo metateórico que Moulines suscribe, y al que ha hecho aportes sustanciales, es un heredero directo de la fase clásica de la filosofía de la ciencia. Como tal, mantiene las teorías en el lugar central de su enfoque (si bien ha ampliado la noción clásica elaborando el concepto de red teórica) y todavía busca ofrecer la mejor "reconstrucción racional" de estos productos de la ciencia. Este punto de vista, en mi opinión, lleva a los estructuralistas a sobrevalorar la importancia de las teorías científicas y a descuidar todas las prácticas científicas que no están al servicio de las teorías. Incluso la noción misma de modelo es para algunos epistemólogos de finales del siglo XX (por ejemplo, para Morgan y Morrison 1999) independiente de la de teoría; la construcción de modelos, por consiguiente, es una actividad que no puede capturarse mediante el estudio de las teorías. Este enfoque se ha reforzado durante la primera década del siglo XXI, hasta el punto de que muchos filósofos de la ciencia han prácticamente remplazado la noción de teoría por la de modelo, a la vez que han privilegiado el estudio de la actividad de construir modelos (la "estrategia del modelador") por sobre el análisis de los modelos mismos, entendidos como productos y vehículos del conocimiento científico. Ya a finales de la década de 1980, Hacking diagnosticaba que la filosofía de la ciencia se había "empobrecido" por su "obsesión con la teoría" (Hacking 1989, p. 148). En los últimos años de siglo XX los problemas de la estructura y la dinámica de las teorías habían dejado de ser prioritarios en la agenda de la mayoría de los filósofos de la ciencia. Actualmente muy pocos filósofos estarían dispuestos a 
conceder a las teorías el lugar privilegiado que la filosofía clásica de la ciencia les había dado y que Moulines todavía conserva. Como resultado de su inclinación "teoricista", el relato que nos ofrece Moulines casi no deja lugar para las prácticas científicas ni, más en general, para el estudio de la ciencia como proceso. Hoy en día, muchos filósofos piensan, en cambio, que las teorías sólo son uno entre los muchos productos de la ciencia y que el análisis de los procesos de la investigación científica es epistemológica y filosóficamente tan relevante como el de sus productos.

\section{Otros enfoques posibles}

No quiero dar la impresión de que critico a Moulines por adoptar un punto de vista a partir del cual selecciona e interpreta sus datos históricos. No hay nada de objetable en ello, puesto que de otro modo la historiografía sería imposible. Es obvio que todo relato histórico es el resultado de un complejo proceso de selección, interpretación y valoración de las fuentes en el que resulta difícil separar cada uno de esos componentes, íntimamente imbricados entre sí. Sería, por tanto, ingenuo pensar que existe un relato correcto o privilegiado de la historia. Hay siempre varios relatos diferentes compatibles con toda la información disponible en un momento dado, del mismo modo que hay muchas teorías posibles capaces de acomodar toda la evidencia conocida. Además, las diferencias de selección, valoración e interpretación se reflejarán inevitablemente en narrativas diferentes, aunque no necesariamente incompatibles entre sí. Sólo quiero señalar ahora otros puntos de vista que podrían adoptarse para relatar la historia de la filosofía de la ciencia, sobre todo la más reciente, caracterizada por la pluralidad y la diversidad más que por el predominio de alguna escuela o corriente determinada.

La discusión entre realismo y antirrealismo, por ejemplo, proporciona un eje temático ortogonal al de la concepción semántica de las teorías (de hecho, algunos de sus miembros más destacados, como Giere y van Fraassen, se ubican en bandos diferentes del debate). Esta temática constituye, además, un hilo conductor alternativo que permitiría trazar un mapa de la filosofía de la ciencia de las tres últimas décadas del siglo XX muy amplio y comprehensivo, tanto en temas como en autores. Caben allí todas las discusiones sobre la subdeterminación de las teorías por la evidencia (desde Quine 1975, hasta Laudan y Leplin 1991); la metainducción pesimista (Laudan 1981); el argumento realista de la explicación del éxito predictivo (Boyd 1983); las teorías formales de la verosimilitud (Oddie 1986 y 
Niiniluoto 1987); el empirismo constructivo de van Fraassen (1980 y 1989); los realismos debilitados, como el realismo interno de Putnam (1978) (que, en mi opinión, es una forma de convencionalismo), la actitud ontológica natural de Fine (1984), el realismo estructural de Worrall (1989) y otros; las teorías de la referencia directa para los términos teóricos desde Putnam (1973) en adelante, entre tantos otros. Moulines menciona algunos de estos temas en el último parágrafo de su libro (pp. 158-165) pero resulta demasiado breve como para hacer justicia a la riqueza de tales cuestiones.

El debate entre realistas y antirrealistas, que probablemente no admita solución definitiva, como la mayoría de los problemas genuinamente filosóficos, tiene también otras implicaciones más generales que me parecen particularmente importantes para la filosofía de la ciencia. En el fondo, el desacuerdo entre los diferentes participantes de este debate es un desacuerdo acerca de cuáles son, o deberían ser, los fines últimos del conocimiento científico y de la ciencia como actividad humana. Plantearse esta pregunta implica, inevitablemente, adentrarse en la difícil cuestión de los valores epistémicos y de la manera en que éstos se relacionan entre sí y con otros valores no epistémicos que también pueden intervenir en la orientación de la práctica de la ciencia y en la delimitación de sus objetivos mismos. Son cuestiones que todavía aguardan un análisis sistemático y detallado, pero que, sin duda alguna, influyeron en el desarrollo de toda la filosofía de la ciencia desde sus orígenes.

La filosofía de la experimentación (incluyendo allí a la de la observación científica) es, en mi opinión, otro de los ejes característicos de la filosofía general de la ciencia de las dos últimas décadas del siglo XX. Hay que contar, entre otros estudios, la antropología de laboratorio de Latour y Woolgar (1979) y los desafíos sociológicos de Collins (1985) y Gooding (1990). La obra pionera en filosofía de la experimentación es la de Hacking (1983), la única a la que Moulines dedica un comentario (pp. 147-151), y luego le siguen la epistemología de la experimentación de Franklin (1986 y 1990), los trabajos histórico-filosóficos de Galison (1987 y 1997) y la filosofía de la confirmación experimental de Mayo (1996). Todas estas obras renovaron completamente el campo, hasta entonces casi olvidado, de la filosofía de la experimentación y mostraron la multiplicidad de funciones que cumple la ciencia experimental, en modo alguno limitada a la confirmación de teorías. Paralelamente, se produjeron los primeros estudios serios acerca de la observación en la ciencia, a partir del artículo pionero de Shapere (1982). Luego, Brown (1987) y Kosso (1989) desarrollaron con detalle una teoría causal-informacional de 
la observación mucho más adecuada a la práctica científica que el estrecho esbozo de análisis de los empiristas lógicos. Además, el surgimiento de los estudios históricos y sociales sobre la experimentación tuvo una influencia decisiva en el vuelco de la filosofía de la ciencia hacia las prácticas científicas y los procesos de producción del conocimiento. Así pues, la filosofía de la observación y la experimentación proporciona otro hilo conductor alternativo, y de suma importancia en mi opinión, para comprender el desarrollo de la filosofía de la ciencia, sobre todo en las últimas dos décadas del siglo XX y hasta nuestros días.

Un enfoque diferente es el que toma en cuenta el desarrollo y la función de las instituciones académicas. Al comienzo de su libro Moulines se refiere a los "inicios institucionales" de la filosofía de la ciencia en el siglo XIX (p. 11), pero luego no prosigue ese hilo conductor. Creo que vale la pena explorarlo ya que con frecuencia, una disciplina científica está modelada en mucho mayor medida por estas instituciones que por la obra de los grandes científicos. La filosofía de la ciencia no es una excepción. No cabe duda de que la creación del Círculo de Viena, la aparición de la revista Erkenntnis y la organización periódica de los Congresos para la Unidad de la Ciencia contribuyeron de manera decisiva a la creación de la filosofía de la ciencia como una disciplina autónoma. Después de la emigración masiva de los filósofos de lengua alemana, el centro productor de la filosofía de la ciencia se trasladó a Estados Unidos de América, y en menor medida a Gran Bretaña. Luego de finalizada la Segunda Guerra Mundial, la filosofía clásica de la ciencia se halla bien establecida en Estados Unidos, pero mucho menos en los países de Europa continental, donde encuentra resistencias institucionales casi hasta el fin del siglo XX. La agenda de los temas en discusión la establece principalmente la revista Philosophy of Science, donde se publican muchos trabajos clásicos de Carnap, Hempel y otros filósofos. Contribuyen también los volúmenes de los Minnesota Studies in the Philosophy of Science y los Boston Studies in the Philosophy of Science. Luego, se funda la Philosophy of Science Association (PSA) que organiza congresos anuales donde se reúnen los filósofos de la ciencia más destacados. Incluso hasta finales del siglo, estas instituciones marcan la tendencia de la corriente principal de la filosofía académica de la ciencia. La lengua inglesa se vuelve casi excluyente como vehículo de comunicación de los resultados originales de investigación, como ya había ocurrido en las ciencias naturales, y las lenguas nacionales como el alemán y el francés declinan notablemente. Las ciencias naturales, comenzando por la física y siguiendo por la biología, tienen una 
prioridad evidente en la agenda de estas instituciones, hasta el punto de que las ciencias sociales quedan marginadas o directamente excluidas (por ejemplo, de las páginas de Philosophy of Science y de los congresos de la PSA). Mucho tiempo después, se organizan las instituciones europeas, comenzando por la prestigiosa British Journal for the Philosophy of Science. Luego aparecen otras revistas europeas, pero ya no de carácter nacional ni asociadas a una universidad, sino a grupos comerciales, como Synthese y la nueva versión de Erkenntnis (relanzada en 1975, pero que nunca alcanzó la importancia de la original), en las cuales la lengua inglesa es también la dominante. La organización de congresos e instituciones académicas llega mucho más tarde.

La filosofía general de la ciencia gradualmente se consolida como una asignatura que se considera parte de la formación básica de cualquier filósofo, a la par de las disciplinas tradicionales como la teoría del conocimiento, la metafísica y la ética. Éste es un proceso que tiene un desarrollo muy desigual en diferentes países y regiones; se consuma con relativa rapidez en los países de lengua inglesa, principalmente en Inglaterra y Estados Unidos, y lo hace de manera más lenta y tardía en las regiones de habla española, portuguesa o francesa. Paralelamente, desde principios de la década de 1950 comienza la producción de libros de texto y de antologías de trabajos originales destinadas a cursos de grado y posgrado, como la conocida compilación de Feigl y Brodbeck (1953), utilizada durante décadas. Poco después, los libros de texto de carácter introductorio se multiplican sin pausa, siguiendo el modelo de la obra de Hempel (1966), de ejemplar claridad. A ello se añade la producción, más reciente, de léxicos, companions y enciclopedias que, sobre todo, recopilan información y, en general, no ofrecen un punto de vista unificado, por el hecho particular de estar conformadas por el agregado de entradas escritas por diferentes autores. ${ }^{14}$ A partir de la década de 1990, pero aún más en la primera década del siglo XXI, la elaboración de antologías de trabajos originales, generalmente comentados, se convierte en una tarea que hoy constituye toda una industria académica, principalmente en Estados Unidos. ${ }^{15}$ Son raros, en cambio, los tratados avanzados y sistemáticos,

${ }^{14}$ Entre otras, pueden citarse las compilaciones Newton-Smith 2000, Sarkar y Pfeifer 2005 y Curd y Psillos 2008.

${ }^{15}$ Por ejemplo, las compilaciones Boyd, Gasper y Trout 1991; Cover y Curd 1998; Balashov y Rosenberg 2002, y Lange 2007, entre muchas otras semejantes. Casi todas tienen coincidencias sustanciales en la selección de temas, autores e incluso textos específicos. McGrew, Alspector-Kelly y Allhoff 2009 es una de las pocas que incluye a los filósofos y científicos anteriores al siglo XX. 
al estilo del clásico de Nagel (1961), uno de los últimos en su género, que ya no podrían tener un único autor, dada la diversidad y fragmentación del conocimiento actualmente imperantes en la disciplina. Los tratados generales son, ya desde la década de 1990, obras colectivas. ${ }^{16}$ Toda esta proliferación bibliográfica merece tenerse en cuenta en una historia de la filosofía de la ciencia. Aunque pueda parecer externa a la historia de las ideas, proporciona información importante acerca de cuáles son los temas que en un momento dado se consideran establecidos en la agenda de los filósofos de la ciencia. Además, ese tipo de textos contribuye a conformar la matriz disciplinar en la cual se educan las sucesivas generaciones de filósofos de la ciencia profesionales. Por último, también intervienen, a veces de manera decisiva, en la formación del canon filosófico, es decir, en la determinación de las obras y autores que se considerarán clásicos en la disciplina. Hay, en suma, una rica historia institucional de la filosofía de la ciencia que todavía no ha sido relatada en casi ninguna de sus etapas. ${ }^{17}$

Por último, me parece claro que a partir de la década de 1970 la mayoría de los desarrollos más amplios e importantes de la filosofía de la ciencia se produjeron en el dominio de la filosofía de las ciencias específicas, primero de la física y luego de la biología. Estas dos disciplinas absorbieron los esfuerzos de la mayor parte de los filósofos profesionales de la ciencia (incluso los de quienes también hicieron contribuciones importantes a la filosofía general, como Kitcher 2003 y van Fraassen 1991). Por consiguiente, una historia de la filosofía general de la ciencia será inevitablemente parcial e incompleta. La filosofía especial de la ciencia no se desarrolló de manera independiente de la filosofía general, sino que interactuó con ella de maneras múltiples y complejas que todavía no se han estudiado. No es improbable conjeturar que muchas propuestas recientes sobre cuestiones de filosofía general de la ciencia, como los modelos de explicación, se vieron influidas de manera decisiva por otros avances previos en la filosofía de la física o la biología. ${ }^{18}$ La comprensión del desarrollo de la filosofía de la ciencia, en mi opinión, requiere una visión de conjunto de la filosofía general y especial. Ésta es una tarea mayor que seguramente ocupará a las futuras generaciones de filósofos pero que en la actualidad apenas ha comenzado.

\footnotetext{
${ }^{16}$ Entre otras, las compilaciones Salmon y Salmon 1992, y Kuipers 2007.

${ }^{17}$ Reisch 2005 es un intento, en mi opinión relativamente poco logrado, de abordar la historia de la filosofía de la ciencia con este enfoque.

${ }^{18}$ Por supuesto, esta conjetura debería confirmarse mediante el análisis de casos concretos.
} 


\section{BIBLIOGRAFÍA}

Balashov, Y. y A. Rosenberg (comps.), 2002, Philosophy of Science: Contemporary Readings, Routledge, Nueva York.

Boyd, R., 1983, "On the Current Status of Scientific Realism", Erkenntnis, vol. 19, pp. 45-90.

Boyd, R., P. Gasper y J. Trout (comps.), 1991, The Philosophy of Science, The MIT Press, Cambridge, Mass.

Bridgman, P., 1927, The Logic of Modern Physics, Macmillan, Nueva York. Brown, H.I., 1987, Observation and Objectivity, Oxford University Press, Nueva York.

Campbell, N.R., 1921, What is Science?, Methuen, Londres.

- 1920, Physics: The Elements, Cambridge University Press, Cambridge.

Carnap, R., 1956, "The Methodological Character of Theoretical Concepts", en H. Feigl y M. Scriven (comps.), Minnesota Studies in the Philosophy of Science, University of Minnesota Press, Mineápolis, 1956, vol. I, pp. 38-76.

- 1950, Logical Foundations of Probability, The University of Chicago Press, Chicago.

- 1936-1937, "Testability and Meaning", Philosophy of Science, vol. 3, pp. 420-468 y vol. 4, pp. 1-40.

—_, 1928, Der logische Aufbau der Welt, Weltkreis, Berlín.

Cassini, A., 2010, "Tiene algún futuro la filosofía general de la ciencia", en A. Cassini y L. Skerk (comps.), Presente y futuro de la filosofía, Editorial de la Facultad de Filosofía y Letras-Universidad de Buenos Aires, Buenos Aires, pp. 81-109.

— 2009, “Son necesarios los modelos para identificar a las teorías? Una crítica de la concepción semántica", en C.A. Mortari y L.H. de A. Dutra (comps.), Anais do V Simpósio Internacional Principia, Núcleo de Epistemologia e Lógica-Universidade Federal de Santa Catarina, Florianópolis (Rumos da Epistemología, 9), pp. 23-37.

Cohen, M.R. y E. Nagel, 1934, An Introduction to Logic and Scientific Method, Harcourt Brace, Nueva York.

Collins, H., 1985, Changing Order: Replication and Induction in Scientific Practice, Sage, Londres.

Cover, J. y M. Curd (comps.), 1998, Philosophy of Science: The Central Issues, Norton, Nueva York/Londres.

Craig, W., 1956, "Replacement of Auxiliary Expressions", Philosophical Review, vol. 65, no. 1, pp. 38-55.

— 1953, "On Axiomatizability within a System", Journal of Symbolic Logic, vol. 18, no. 1, pp. 30-32.

Curd, M. y S. Psillos (comps.), 2008, The Routledge Companion to the Philosophy of Science, Routledge, Londres/Nueva York. 
Díez, J.A. y C.U. Moulines, 2008, Fundamentos de filosofía de la ciencia, 3a. ed., Ariel, Barcelona. [Primera edición, 1997.]

Duhem, P., 1906, La Théorie physique: son objet, sa structure, Chevalier et Rivière, París.

Feigl, H. y M. Bordbeck (comps.), 1953, Readings in the Philosophy of Science, Appleton-Century-Crofts, Nueva York.

Fine, A., 1993, "Fictionalism", Midwest Studies in Philosophy, vol. 18, no. 1, pp. 1-18.

— , 1984, "The Natural Ontological Attitude", en J. Leplin (comp.), Scientific Realism, University of California Press, Berkeley, pp. 83-107.

Franklin, A., 1990, Experiment: Right or Wrong, Cambridge University Press, Cambridge.

- - 1986, The Neglect of Experiment, Cambridge University Press, Cambridge.

Galison, P., 1997, Image and Logic: A Material Culture of Microphysics, The University of Chicago Press, Chicago.

- 1987 , How Experiments End, The University of Chicago Press, Chicago.

Gooding, D., 1990, Experiment and the Making of Meaning: Human Agency in Scientific Observation and Experiment, Kluwer, Dordrecht.

Goodman, N., 1951, The Structure of Appearance, Harvard University Press, Cambridge, Mass.

Gower, B., 1997, Scientific Method: A Historical and Philosophical Introduction, Routledge, Londres.

Hacking, I., 1989, "Philosophers of Experiments", PSA: Proceedings of the Biennial Meeting of the Philosophy of Science Association, vol. 2, pp. 147-156.

- 1983 , Representing and Intervening: Introductory Topics in the Philosophy of Natural Science, Cambridge University Press, Cambridge.

Hanson, N.R., 1958, Patterns of Discovery: An Inquiry into the Conceptual Foundations of Science, Cambridge University Press, Cambridge.

Hempel, C.G., 2001, The Philosophy of Carl G. Hempel: Studies in Science, Explanation, and Rationality, ed. James H. Fetzer, Oxford University Press, Nueva York.

—_, 1966, Philosophy of Natural Science, Prentice Hall, Englewood Cliffs.

- 1965, Aspects of Scientific Explanation and Other Essays in the Philosophy of Science, The Free Press, Nueva York.

— , 1958, "The Theoretician's Dilemma", en H. Feigl, M. Scriven y G. Maxwell (comps.), Minnesota Studies in the Philosophy of Science, University of Minnesota Press, Minnesota, vol. II, pp. 37-98. [Reimpreso en Hempel 1965, pp. 173-226.]

-, 1952, Fundamentals of Concept Formation in Empirical Science, The University of Chicago Press, Chicago. 
Hempel, C.G., 1945, "Studies in the Logic of Confirmation", Mind, vol. 54, no. 213, pp. 1-16, y no. 214, pp. 97-121. [Reimpreso, con un "Postscript (1964) on Confirmation", en Hempel 1965, pp. 3-51.]

Herschel, J., 1830, A Preliminary Discourse on the Study of Natural Philosophy, John Taylor, Londres.

Jevons, W.S., 1874, The Principles of Science: A Treatise on Logic and Scientific Method, MacMillan, Londres.

Kitcher, P., 2003, In Mendel's Mirror: Philosophical Reflections on Biology, Oxford University Press, Nueva York.

Kosso, P., 1989, Observability and Observation in Physical Science, Kluwer, Dordrecht.

Kuhn, T.S., 2000, The Road since Structure: Philosophical Essays, 19701993, with an Autobiographical Interview, The University of Chicago Press, Chicago.

- 1984, "Revisiting Planck", Historical Studies in the Physical Sciences, vol. 14, no. 2, pp. 231-252.

—_ 1978, Black Body Theory and the Quantum Discontinuity, 18941912, The University of Chicago Press, Chicago.

— , 1970, "Reflections on My Critics", en I. Lakatos y A. Musgrave (comps.), Criticism and the Growth of Knowledge, Cambridge University Press, Cambridge, pp. 231-278.

- 1962, The Structure of Scientific Revolutions, The University of Chicago Press, Chicago.

Kuipers, T. (comp.), 2007, General Philosophy of Science: Focal Issues, Elsevier, Amsterdam.

Lange, M. (comp.), 2007, Philosophy of Science: An Anthology, Blackwell, Oxford.

Latour, B. y S. Woolgar, 1979, Laboratory Life: The Social Construction of Scientific Facts, Sage, Londres.

Laudan, L., 1981, “A Confutation of Convergent Realism”, Philosophy of Science, vol. 48, no. 1, pp. 19-48.

Laudan, L. y J. Leplin, 1991, "Empirical Equivalence and Underdetermination", The Journal of Philosophy, vol. 88, pp. 449-472.

Losee, J., 2001, A Historical Introduction to the Philosophy of Science, 4a. ed., Oxford University Press, Nueva York. [Primera edición, 1972.]

Mayo, D., 1996, Error and the Growth of Experimental Knowledge, The University of Chicago Press, Chicago.

McGrew, T., M. Alspector-Kelly y F. Allhoff (comps.), 2009, Philosophy of Science: An Historical Anthology, Wiley-Blackwell, Oxford.

McKinsey, J.J., A.C. Sugar y P. Suppes, 1953, "Axiomatic Foundations of Classical Particle Mechanics", Journal of Rational Mechanics and Analysis, vol. 2, no. 2, pp. 253-272.

Meyerson, É., 1908, Identité et realité, Felix Alcan, París.

Mill, J.S., 1843, A System of Logic: Ratiocinative and Inductive, John W. Parker, Londres. 
Morgan, M. y M. Morrison, 1999, Models as Mediators: Perspectives on Natural and Social Science, Cambridge University Press, Cambridge.

Moulines, C.U., 2008, Die Entwicklung der modernen Wissenschaftheorie (1890-2000): Eine historische Einführung, Lit, Hamburgo.

—_ 2006, La Philosophie des sciences: l'invention d'une discipline (fin $X I X^{e}$-début $X X I^{e}$ siècle), Rue d'Ulm, París.

Nagel, E., 1961, The Structure of Science: Problems in the Logic of Scientific Explanation, Harcourt, Brace and World, Nueva York.

Newton-Smith, W.H., 2000, A Companion to the Philosophy of Science, Blackwell, Oxford.

Niiniluoto, I., 1987, Truthlikeness, Reidel, Dordrecht.

Oddie, G., 1986, Likeness to Truth, Reidel, Dordrecht.

Oldroyd, D., 1986, The Arch of Knowledge: An Introductory Study of the History of the Philosophy and Methodology of Science, Methuen, Nueva York.

Pearson, K., 1892, The Grammar of Science, Walter Scott, Londres.

Pickering, A. (comp.), 1992, Science as Practice and Culture, The University of Chicago Press, Chicago.

Poincaré, H., 1902, La Science et l'hypothèse, Flammarion, París.

Popper, K.R., 1994, The Myth of the Framework: In Defence of Science and Rationality, ed. M.A. Notturno, Routledge, Nueva York.

- - 1934, Logik der Forschung, Springer, Viena. [Edición inglesa ampliada: The Logic of Scientific Discovery, Hutchinson, Londres, 1959.]

Putnam, H., 1978, Meaning and the Moral Sciences, Routledge and Kegan Paul, Londres.

—_, 1973, "Explanation and Reference", en G. Pearce y P. Maynard (comps.), Conceptual Change, Reidel, Dordrecht, pp. 199-221.

Quine, W.V.O., 1975, "On Empirically Equivalent Systems of the World", Erkenntnis, vol. 9, pp. 313-328.

- 1951, "Two Dogmas of Empiricism", Philosophical Review, vol. 60, no. 1 , pp. 20-43.

Reichenbach, H., 1951, The Rise of Scientific Philosophy, University of California Press, Berkeley.

—_ 1938, Experience and Prediction: An Analysis of the Foundations and the Structure of Knowledge, The University of Chicago Press, Chicago.

Reisch, G., 2005, How the Cold War Transformed Philosophy of Science: To the Icy Slopes of Logic, Cambridge University Press, Cambridge.

Rubin, H. y P. Suppes, 1954, "Transformations of Systems of Relativistic Particle Mechanics", Pacific Journal of Mathematics, vol. 4, no. 4, pp. 563-601.

Salmon, W. y M. Salmon (comps.), 1992, Introduction to the Philosophy of Science, Prentice Hall, Englewood Cliffs.

Sarkar, S. y J. Pfeifer (comps.), 2005, Philosophy of Science: An Encyclopedia, Routledge, Londres, 2 vols. 
Scheffler, I., 1967, Science and Subjectivity, Bobbs Merrill, Indianápolis.

Schlick, M., 1918, Allgemeine Erkenntnislehre, Springer, Berlín.

Shapere, D., 1982, "The Concept of Observation in Science and Philosophy", Philosophy of Science, vol. 49, no. 4, pp. 485-525.

Stadler, F., 1997, Studien zum Wiener Kreis: Ursprung, Entwicklung und Wirkung des Logischen Empirismus im Kontext, Suhrkamp, Fráncfort del Meno.

Stegmüller, W., 1979, The Structuralist View of Theories, Springer, Berlín. Suppe, F., 2000, "Understanding Scientific Theories: An Assessment of Developments, 1969-1998", Philosophy of Science, vol. 67, no. 3, pp. S102-S115.

Suppes, P., 2002, Representation and Invariance of Scientific Structures, Center for the Study of Language and Information, Stanford.

—_, 1967, "What is a Scientific Theory?", en S. Morgenbesser (comp.), Philosophy of Science Today, Basic Books, Nueva York, pp. 55-67.

—_, 1957, Introduction to Logic, Van Nostrand, Nueva York.

Toulmin, S., 1961, Foresight and Understanding, Hutchinson, Londres. Londres.

Vaihinger, H., 1911, Die Philosophie des Als Ob, Reuther und Reichard, Berlín. [Décima edición: Felix Meiner, Leipzig, 1927.]

Van Fraassen, B.C., 1991, Quantum Mechanics: An Empiricist View, Clarendon Press, Oxford.

—., 1989, Laws and Symmetry, Clarendon Press, Oxford.

—_, 1980, The Scientific Image, Clarendon Press, Oxford.

Weyl, H., 1927, Philosophie der Mathematik und Naturwissenschaft, Oldenbourg, Múnich.

Whewell, W., 1840, The Philosophy of the Inductive Sciences, John W. Parker, Londres.

Worrall, J., 1989, “Structural Realism: The Best of Both Worlds?", Dialectica, vol. 43, nos. 1-2, pp. 99-124.

Recibido el 11 de mayo de 2012; revisado el 16 de agosto de 2012; aceptado el 7 de septiembre de 2012. 